\title{
CAFI-knockout mice are more susceptive to lipopolysaccharide-induced acute lung injury
}

This article was published in the following Dove Press journal:

Journal of Inflammation Research

II June 2016

Number of times this article has been viewed

\begin{abstract}
Jia-Xin Shi ${ }^{1}$
Jia-Shu Li'

Rong $\mathrm{Hu}^{\prime}$

Xiao-Min $\mathrm{Li}^{2}$

Hong Wang ${ }^{3}$

'Department of Respiratory Medicine, Lianyungang First People's Hospital, Affiliated Hospital of Xuzhou

Medical University, Affiliated Hospital of the Clinical Medical School of Nanjing Medical University, Clinical Medical School of Jiangsu University, Lianyungang, ${ }^{2}$ Department of Critical Care Medicine, Lianyungang First People's Hospital, Affiliated Hospital of Xuzhou Medical University, Affiliated Hospital of the Clinical Medical School of Nanjing Medical University, Clinical Medical School of Jiangsu University, Lianyungang, ${ }^{3}$ Department of Respiratory Medicine, Jiangsu Province Hospital, the First Affiliated Hospital of Nanjing Medical University, Nanjing, People's Republic of China
\end{abstract}

Correspondence: Jia-Xin Shi Department of Respiratory Medicine, Lianyungang First People's Hospital, Affiliated Hospital of Xuzhou Medical University, Affiliated Hospital of Clinical Medical School of Nanjing Medical University, Affiliated Hospital of Clinical Medical School of Jiangsu University, 182 Northern Tongguan Road, Lianyungang 222002, People's Republic of China

Tel +865l8 85605041

$\mathrm{Fax}+8651885456700$
Abstract: The carbon catabolite repressor protein 4 (CCR4)-negative on TATA (NOT) complex includes multiple subunits and is conserved in the eukaryotic cells. The CCR4-NOT complex can regulate gene expression at different levels. Two subunits of the CCR4-NOT complex, CCR4 and CCR4-associated factor $1(C A F 1)$, possess deadenylase activity. In yeast, the deadenylase activity is mainly provided by the CCR4 subunit; however, the deadenylase activity is provided by both CCR 4 and $C A F 1$ in other eukaryotes. A previous study reported that $C A F 1$ but not CCR4 is required for the decay of a reporter mRNA with AU-rich elements. Our previous study showed that $C A F 1$ is involved in the regulation of intercellular adhesion molecule-1 (ICAM-1) and interleukin-8 (IL-8) expression. Both ICAM-1 and IL-8 play crucial roles in acute lung injury. In the present study, we examined the effects of $C A F 1$ deficiency on IL-8 and ICAM-1 expression and acute lung injury in mice. Here we showed that there were no differences between the wild-type and CAF1-knockout mice on phenotypes. The lung histology and protein and mRNA levels of IL-8 and ICAM-1 in unstimulated wild-type mice were comparable to those in unstimulated $C A F 1$-knockout mice. However, lipopolysaccharide stimulation led to more severe lung histological injury and greatly higher IL-8 and ICAM-1 expression in CAF1-knockout mice compared to the wild-type mice. These results, together with our previous study, suggest that $C A F 1$ is involved in the regulation of lipopolysaccharide-stimulated IL-8 and ICAM-1 expression in vivo and affects the progression of acute lung injury.

Keywords: $C A F 1$, knockout, mice, acute lung injury, IL-8, ICAM-1

\section{Introduction}

The carbon catabolite repressor protein 4 (CCR4)-negative on TATA (NOT) complex includes multiple subunits and is conserved in the eukaryotic cells. The CCR4-NOT complex can regulate gene expression at different levels. ${ }^{1}$ Two subunits of the CCR4NOT complex, CCR4 and CCR4-associated factor $1(C A F 1)$, possess deadenylase activity. In yeast, the deadenylase activity is mainly provided by the CCR4 subunit; however, the deadenylase activity is provided by both CCR4 and CAF1 (also called CNOT7) in other eukaryotes. ${ }^{2}$ Schwede et $\mathrm{al}^{3}$ reported that $C A F 1$ rather than $\mathrm{CCR} 4$ is required for the decay of a reporter mRNA with AU-rich element (ARE) at $3^{\prime}$-untranslated region ( $3^{\prime}$-UTR). ARE-mediated mRNA decay plays an important role in posttranscriptional regulation in inflammatory mediator expression. ${ }^{4}$

Tristetraprolin (TTP) is a tandem zinc-finger RNA-binding protein that can bind to the AREs in $3^{\prime}$-UTR and then lead to mRNA deadenylation and degradation. ${ }^{5} \mathrm{We}$ have shown in a previous study that TTP regulates intercellular adhesion molecule-1 submit your manuscript | www.dovepress.con Dovepress http://dx.doi.org/10.2147/JIR.S105193 
(ICAM-1) and interleukin-8 (IL-8) expression by destabilizing their mRNAs in human pulmonary microvascular endothelial cells, ${ }^{6}$ and then we demonstrated that TTP interacts with CNOT1, the scaffold subunit of the CCR4-NOT complex, to recruit $C A F 1$ and thereby induces degradation of ICAM-1 and IL-8 mRNA. ${ }^{7}$ A previous study has also shown that TTP could bind to CNOT1 which then recruits $C A F 1$ to target mRNA. ${ }^{8}$ Both ICAM-1 and IL-8 play important roles in acute inflammation with polymorphonuclear neutrophil (PMN) chemotaxis, adhesion, and infiltration into the lung tissue during acute lung injury. ${ }^{9}$ Subsequently, overactivation of PMN can release myeloperoxidase (MPO) and then induce lung tissue damage.

Although $C A F 1$ plays an important role in the regulation of ICAM-1 and IL-8 expression in vitro, whether $C A F 1$ could regulate ICAM-1 and IL-8 expression in vivo and eventually affect acute lung injury remains unknown. In the present study, we examined the effects of CAF1 deficiency on IL-8 and ICAM-1 expression and acute lung injury in mice. Here we report that the lung histology, MPO activity, and protein and mRNA levels of IL- 8 and ICAM-1 in unstimulated wild-type mice were comparable to those in unstimulated CAF1-knockout mice. However, lipopolysaccharide (LPS) stimulation led to more severe lung histological injury, higher MPO activity, and greatly higher IL- 8 and ICAM-1 expression in CAF1-knockout mice compared to the wild-type mice. These results, together with our previous study, suggest that $C A F 1$ is involved in the regulation of LPS-stimulated IL-8 and ICAM-1 expression in vivo and affects the progression of acute lung injury.

\section{Materials and methods Materials}

LPS (Cat No L2630) was purchased from Sigma-Aldrich (St Louis, MO, USA). MPO test kit (Cat No A044) was purchased from Nanjing Jiancheng Bioengineering Institute (Nanjing, People's Republic of China). TRIzol reagent (Cat No 15596-026) was obtained from Thermo Fisher Scientific (Waltham, MA, USA). The lysis buffer for Western blotting or immunoprecipitation (IP) (Cat No P0013) and the 5-Bromo-4-Chloro-3-Indolyl Phosphate (BCIP)/nitroblue tetrazolium (NBT) Alkaline Phosphatase Color Development Kit (\#C3206) were purchased from Beyotime Institute of Biotechnology (Nantong, People's Republic of China). Protease inhibitors (\#R1321) were obtained from Fermentas UAB (Vilnius, Lithuania). RiboLock RNase Inhibitors (\#E00381) were obtained from Thermo Fisher Scientific. Protein assay reagent (Cat No KGPBCA) was obtained from Keygen Biotec (Nanjing, People's Republic of China). RevertAid First Strand cDNA Synthesis Kit (\#K1622) was obtained from Fermentas UAB. SYBR Green PCR Master Mix (Cat No 04913850001) was obtained from Hoffman-La Roche Ltd (Basel, Switzerland). A mouse IL-8 enzymelinked immunosorbent assay (ELISA) Kit (\#EMC104) was purchased from Neobioscience (Shenzhen, People's Republic of China). The antibodies used were as follows: mouse anti-CNOT7 (CAF1) antibody (sc-101009) was purchased from Santa Cruz Biotechnology Inc. (Dallas, TX, USA), goat anti-ICAM-1 antibody (\#AF796) was purchased from R\&D Systems, Inc. (Minneapolis, MN, USA), and mouse anti- $\beta$-tubulin antibody (\#M20005) was purchased from Abmart (Shanghai, People's Republic of China).

\section{Generation of CAFI-knockout mice}

The gene-knockout mice were generated according to the procedure of a previous study. ${ }^{10}$

\section{Production of Cas9 mRNA and sgRNA}

T7 promoter was added to Cas9 coding region by polymerase chain reaction (PCR) amplification using primer Cas9 $\mathrm{F}$ and R. T7-Cas9 PCR product was gel purified and used as the template for in vitro transcription (IVT) using mMESSAGE mMACHINE T7 ULTRA Kit (Thermo Fisher Scientific). Vector pUC57-T7-gRNA was digested by Bsa I. CAF1S1a(GTC TTG CAA CAA CGC C), CAF1-S1b (ACT ATC AGT ACC AAC TGT TG), CAF1-S2a (TCA TAA AGG TCA GTC CGA GC), and $C A F 1-\mathrm{S} 2 \mathrm{~b}$ (AAC AGG GAG AAT ACC CTC C) were ligated to pUC57-T7-gRNA (Bsa I). Single colonies were picked from each transformation plate and were identified by PCR and sequencing. The sgRNA was transcribed in vitro. Both the Cas9 mRNA and the sgRNAs were purified using MEGA clear kit (Thermo Fisher Scientific) and eluted in RNase-free water.

\section{Zygote microinjection and implantation}

C57BL/6J female mice and B6CBF1 (C57BL/6J $\times$ CBA) mouse strains were used as embryo donors and foster mothers, respectively. Super-ovulated female C57BL/6J mice (3-4 weeks old) were mated with C57BL/6J stud males, and fertilized embryos were collected from oviducts. Cas9 mRNAs (from 20 to $200 \mathrm{ng} / \mathrm{mL}$ ) and sgRNA (from 5 to $50 \mathrm{ng} / \mathrm{mL}$ ) were injected into the cytoplasm of fertilized eggs with well-recognized pronuclei in M2 medium (Sigma-Aldrich). Thereafter, 20-25 fertilized eggs were transferred into the uterus of pseudopregnant B6CBF1 females at $0.5 \mathrm{dpc}$. 
Crossing F0 mice with C57BL/6] mice to build up heterozygous mice $\left(\mathrm{CAFI}^{+} l^{-}\right)$

Crossing the male and female offspring to generate CAFI-I- (CAFI knockout) mice

The genotype of the offspring was determined by PCR and sequencing of tail DNA and TA cloning.

\section{Animals and treatment}

Eight- to 12-week-old CAF1-knockout (n=6) and wildtype mice $(n=6)$ were obtained from the Model Animal Research Center of Nanjing University. Animals were maintained in standard cages in a temperature-controlled room $\left(22^{\circ} \mathrm{C}-25^{\circ} \mathrm{C}\right)$ with a 12 -hour light-dark cycle, with ad libitum access to food and water. The mice model of acute lung injury was produced by intraperitoneal administration of LPS at $15 \mathrm{mg} / \mathrm{kg}$ according to a previous study. ${ }^{11}$ The control mice received normal saline (NS). All the animals were divided into four groups ( $\mathrm{n}=3$ per group): 1 ) wild-type mice with NS, 2) wild-type mice with LPS, 3) CAF1-knockout mice with NS, and 4) CAF1-knockout mice with LPS. The whole blood was collected from mice orbit 8 hours after NS or LPS administration. The mice were humanely sacrificed by cervical dislocation and lungs were removed. All procedures were approved by the Animal Care and Use Committee of the Model Animal Research Center of Nanjing University (Nanjing, People's Republic of China), and all experiments were conducted in accordance with the guidelines set by the Animal Care and Use Committee of the Model Animal Research Center of Nanjing University.

\section{Histopathological examination of lung tissue}

The mice lung tissues were flushed with ice-cold phosphatebuffered saline and slit open longitudinally, and then the lung tissue samples were fixed with $10 \%$ neutral-buffered formalin and embedded in paraffin, cut in 5 - $\mu$ m-thick sections, and then stained with hematoxylin and eosin. The stained sections were examined for histopathological changes by a respiratory pathologist.

\section{Measurements of MPO activity in lung tissues}

The mice lung tissues were homogenized and dissolved in extraction buffer to test MPO activity. In order to analyze the accumulation of PMN, the MPO activity was assessed using MPO assay kit according to the manufacturer's instructions.

\section{Western immunoblotting experiments}

The mice lung tissues were frozen in liquid nitrogen and stored at $-80^{\circ} \mathrm{C}$. The frozen lung samples were homogenized in lysis buffer for Western blotting or IP containing 1\% Triton-X 100 and Protease Inhibitor Cocktail. The procedure of Western immunoblotting was as previously described. ${ }^{7}$ Forty micrograms of total proteins were separated on $10 \%$ Sodium dodecyl sulfate (SDS)-polyacrylamide gels. The primary antibodies (CAFl antibody, ICAM-1 antibody, and $\beta$-tubulin antibody) were diluted at 1:1,000 with phosphate buffer solution containing $0.1 \%$ Tween-20 (PBST) containing 5\% bovine serum albumin. The alkaline phosphatase-conjugated secondary antibodies were diluted at 1:5,000 with PBST containing $5 \%$ bovine serum albumin. The polyvinylidene fluoride (PVDF) membranes were incubated with the BCIP/ NBT Alkaline Phosphatase Color Development Kit until color development was achieved. The band images were acquired by BenQ Scanner (5560C) and analyzed using ImageJ software (http://rsb.info.nih.gov/ij/).

\section{Enzyme-linked immunosorbent assay}

The whole blood was collected from mice orbit and centrifuged at $3,000 \times g$ for 10 minutes, and the blood plasma was collected and stored at $-80^{\circ} \mathrm{C}$. IL-8 levels in blood plasma were measured using an IL-8 ELISA kit according to the manufacturer's instructions. The standard curves were constructed using mouse IL-8 standard. Absorbances were read at $450 \mathrm{~nm}$ using a microplate reader, and the concentrations of IL-8 were calculated according to the standard curves.

\section{RNA isolation, cDNA synthesis, and real-time PCR}

Total RNA was abstracted using TRIzol reagent from the lung samples frozen in liquid nitrogen according to the manufacturer's instructions and synthesized as previously described ${ }^{7}$ and then analyzed with real-time PCR performed using an ABI 7500 Real Time PCR System (Thermo Fisher Scientific). The PCR amplifications were performed using the SYBR Green PCR Master Mix according to the manufacturer's instructions. Glyceraldehyde-3-phosphate dehydrogenase (GAPDH) mRNA was used as the control. Amplification conditions were as follows: $50^{\circ} \mathrm{C}$ for 2 minutes, $95^{\circ} \mathrm{C}$ for 10 minutes, 40 cycles of $95^{\circ} \mathrm{C}$ for 15 seconds, $58^{\circ} \mathrm{C}$ for 30 seconds, and $72^{\circ} \mathrm{C}$ for 30 seconds, followed by melting curve analyses, by which the specificity of primers was confirmed. The primers used for the PCR amplifications are as follows: GAPDH forward, TGC ACC ACC AAC TGC TTA G; 
GAPDH reverse, GAT GCA GGG ATG ATG TTC; ICAM-1 forward, CAA TTT CTC ATG CCG CAC AG; ICAM-1 reverse, AGC TGG AAG ATC GAA AGT CCG; IL-8 forward, CAC CTC AAG AAC ATC CAG AGC T; and IL-8 reverse, CAA GCA GAA CTG AAC TAC CAT CG. $2^{-\Delta \Delta \mathrm{Ct}}$ was used in RNA quantitation.

\section{Statistical analyses}

Statistical analyses were performed by SPSS 19.0 (StataCorp LP, College Station, TX, USA). Differences between the groups were analyzed using an independent sample $t$-test. Two-tailed probability values of $<0.05$ were considered statistically significant. Error bars on images represent standard error of the mean.

\section{Results}

\section{Targeted disruption of the mouse CAFI gene}

The CRISPR/Cas9 gene-knockout technique was applied to disrupt $C A F 1$ gene. The procedure is as described earlier. The mouse $C A F 1$ gene was mutated by deleting the amino acids 128-151 in exon3 (mouse CAFl GenBank accession number U21855) and inserting double "T". Both male and female $\mathrm{CAF}^{-/}{ }^{-}$mice were apparently healthy and exhibited
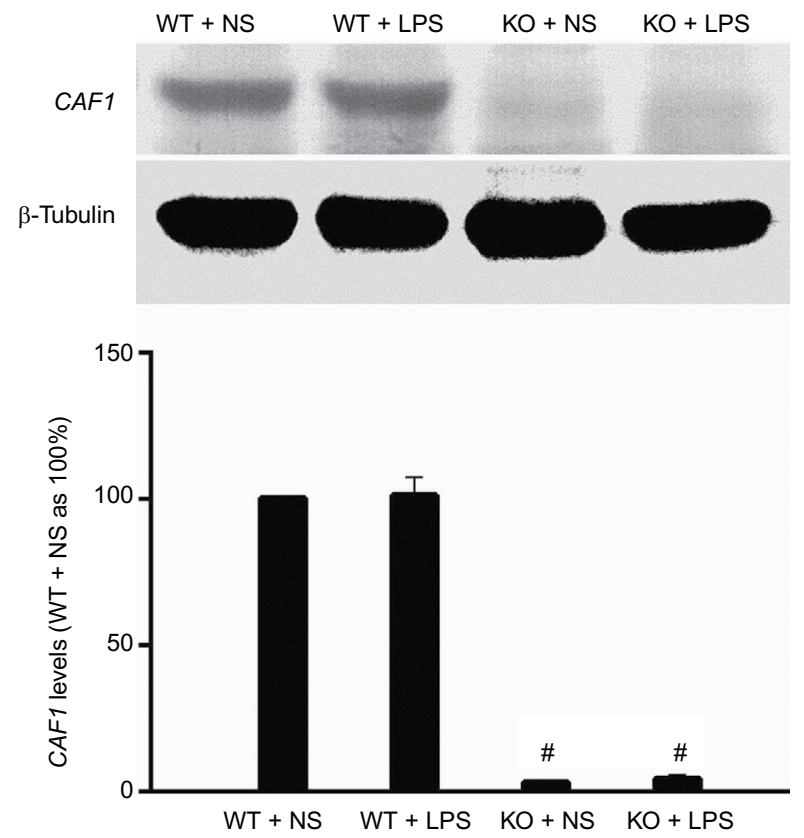

Figure I Mouse CAFI gene knockout abolishes CAFI protein expression. Notes: Western immunoblot band density data showed that CAFI gene knockout greatly reduced CAFI levels and CAFI band was nearly undetectable in CAFIknockout mice. Western immunoblot band density data are expressed as mean \pm SEM. $n=3, \# P<0.05$ vs wild-type mice. An independent sample $t$-test was used. Abbreviations: WT, wild-type mice; KO, CAFI-knockout mice; NS, normal saline; LPS, lipopolysaccharide; SEM, standard error of the mean. no significant abnormalities in external appearance. As shown in Figure 1, compared to the wild-type mice, the $C A F 1$ protein in lung tissue was invisible in $C A F 1^{-/-}$mice, which indicates that $C A F 1$ gene knockout abolishes $C A F 1$ protein expression in vivo.

\section{Histological examination of the lungs of CAFI-1- mice}

The hematoxylin and eosin staining results showed that the lung histology in wild-type mice challenged with NS exhibited normal histological features and was comparable to that in $\mathrm{CAFl}^{-/-}$mice with NS stimulation (Figure 2A and B). In contrast, lungs from wild-type mice with LPS stimulation demonstrated characteristics of acute, diffuse alveolar damage, lots of effusion liquid rich in fiber protein in alveolar space, neutrophil infiltration, and pulmonary edema 8 hours after LPS administration (Figure 2C). Compared to the wild-

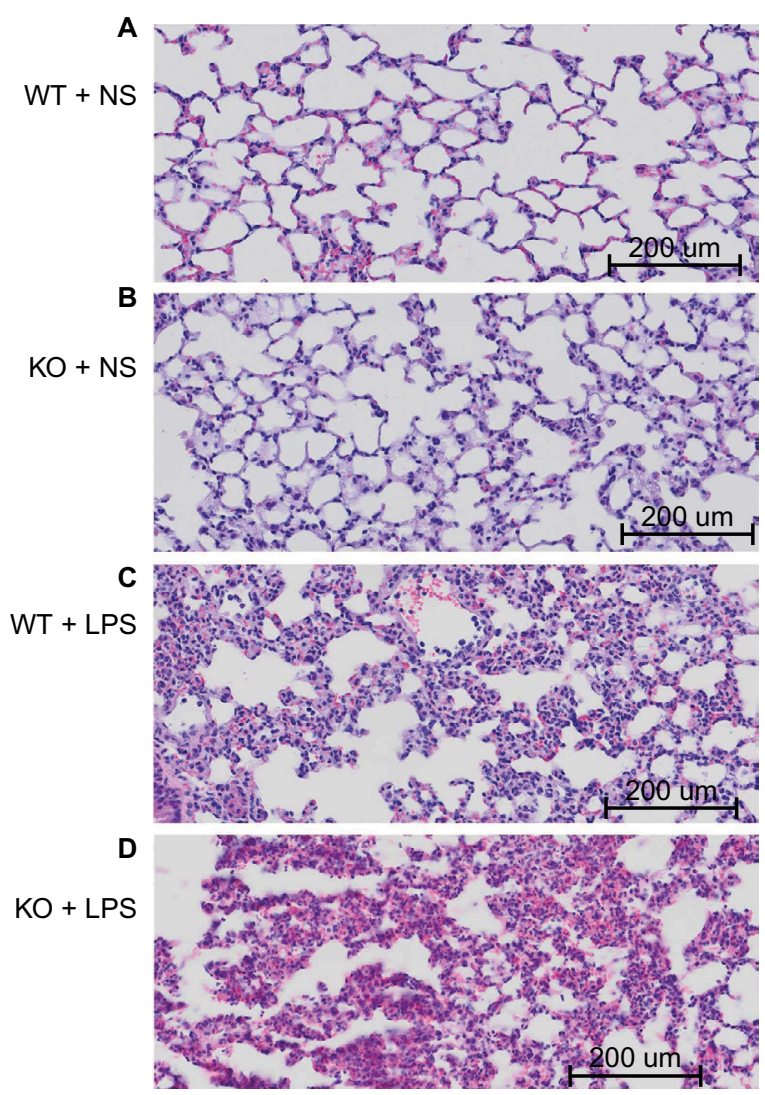

Figure 2 Effect of LPS on lung histology (40x).

Notes: Wild-type and CAFI-knockout mice challenged with NS displayed normal lung histology (A) and (B). On the contrary, lungs from wild-type mice stimulated with LPS exhibited features consistent with acute diffuse lung inflammation (C), which included alveolar and interstitial fluid accumulation, thickened alveolar wall, fibrin effusion, the infiltration of lymphocytes and neutrophils, and the destruction of pulmonary alveoli. These changes were more severe in LPS-stimulated CAFIknockout mice (D).

Abbreviations: WT, wild-type mice; KO, CAFI-knockout mice; NS, normal saline; LPS, lipopolysaccharide. 


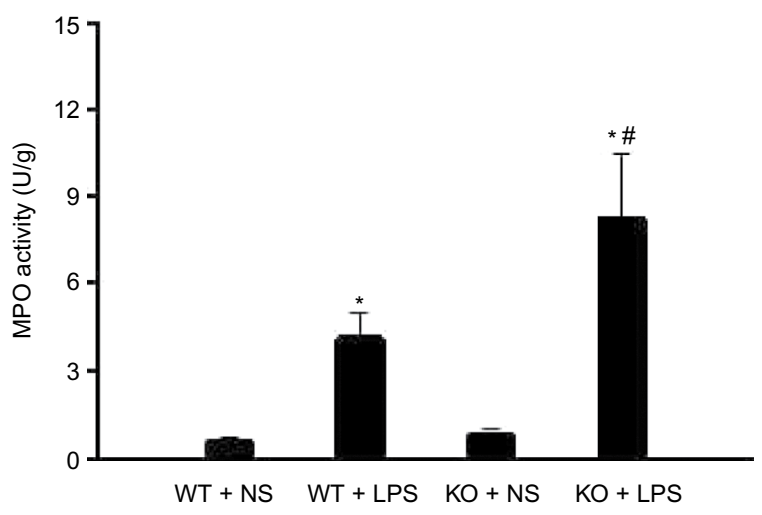

Figure 3 CAFI knockout increases LPS-induced MPO activity.

Notes: MPO test data showed that MPO activity in CAFI-knockout mice treated with NS were comparable to those in wild-type mice. It indicated a significant increase in MPO activity both in wild-type and in CAFI-knockout mice following LPS stimulation, and MPO activity in CAFI-knockout mice was significantly higher than that in wild-type mice. The data are expressed as mean \pm SEM. $n=3, * P<0.05$ vs. NS, $\# P<0.05$ vs. wild-type mice. An independent sample $t$-test was used.

Abbreviations: WT, wild-type mice; KO, CAFI-knockout mice; NS, normal saline; LPS, lipopolysaccharide; MPO, myeloperoxidase.

type mice with LPS stimulation, lungs from $\mathrm{CAF1}^{-/-}$mice challenged with LPS showed more severe alveolar damage, more effusion liquid in alveolar space, and more severe pulmonary edema (Figure 2D). These results indicate that $C A F 1$ deficiency deteriorated the lung histopathologic changes after LPS stimulation.

\section{MPO activity in wild-type and CAFI-I- mice}

Since MPO is mainly released from PMN, we analyzed the MPO activity of the mice lung to indicate the presence of PMN. Wild-type and $\mathrm{CAF1}^{-\gamma^{-}}$mice were treated with NS or LPS for 8 hours, and then the lung tissues were collected for assaying MPO activity. As shown in Figure 3, MPO activity in wild-type mice with NS stimulation was similar to that in $\mathrm{CAF}^{-1^{-}}$mice with NS stimulation. There was a significant increase in MPO activity in $\mathrm{CAF1}^{-/-}$mice treated with LPS compared to the wild-type mice with LPS stimulation.

\section{IL-8 and ICAM-I protein levels in wild-type and $\mathrm{CAFI}^{-I^{-}}$mice}

We reported in a previous study that $C A F 1$ is involved in tumor necrosis-induced ICAM-1 and IL-8 expression in vitro. ${ }^{7}$ However, whether $C A F 1$ could affect ICAM-1 and IL-8 expression in vivo remains unknown. So, we examined the effect of $C A F 1$ deficiency on IL-8 and ICAM-1 expression in vivo. Wild-type and $C A F 1^{-/}$mice were treated with $\mathrm{NS}$ or LPS for 8 hours, and then the blood plasma and total proteins of lung tissues were collected for ELISA and Western immunoblot experiments, respectively. As shown in Figure 4A, IL-8 levels in wild-type mice with NS stimulation were
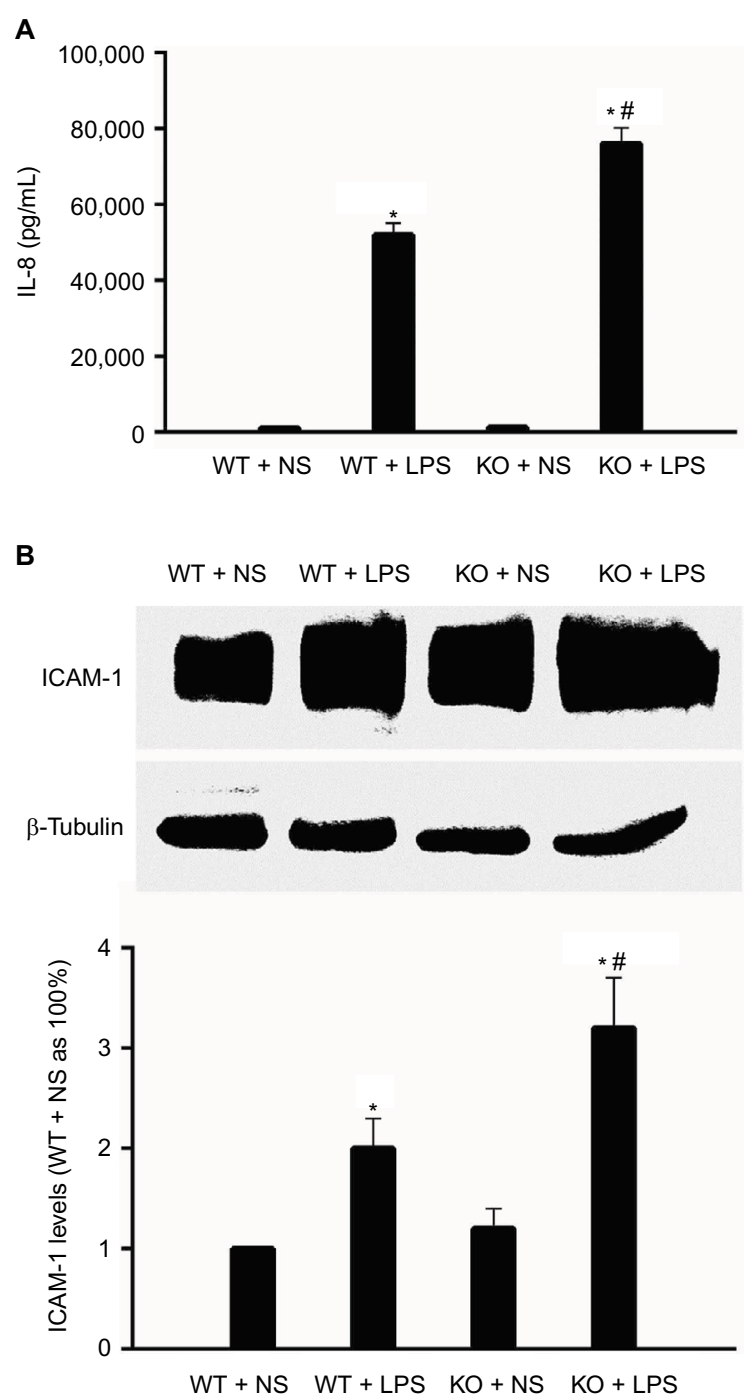

Figure 4 CAFI knockout increases LPS-induced IL-8 and ICAM-I protein levels. Notes: ELISA data indicated IL-8 levels in CAFI-knockout mice challenged with NS were comparable to those in wild-type mice (A). It revealed a great increase in IL-8 both in wild-type and CAFI-knockout mice following LPS stimulation, and IL-8 levels in CAFI-knockout mice were greatly higher than those in wild-type mice (A). Western immunoblot band density data showed that ICAM-I levels in wild-type mice challenged with NS were comparable to those in CAFI-knockout mice (B). LPS stimulation greatly increased ICAM-I expression in both wild-type and $\mathrm{CAFI}^{-1}$ mice compared to NS stimulation. ICAM-I levels in $\mathrm{CAFI}^{-{ }^{-}}$mice stimulated with LPS were greatly more than those in LPS-stimulated wild-type mice (B). ELISA and Western immunoblot band density data are expressed as mean \pm SEM. $n=3, * P<0.05$ vs NS, $\# P<0.05$ vs wild-type mice. An independent sample $t$-test was used.

Abbreviations: WT, wild-type mice; KO, CAFI-knockout mice; NS, normal saline; LPS, lipopolysaccharide; ICAM-I, intercellular adhesion molecule-I; IL-8, interleukin-8; SEM, standard error of the mean; ELISA, enzyme-linked immunosorbent assay.

comparable to those in $C A F 1^{-/-}$mice with NS stimulation. However, there was a significant increase in IL-8 in $\mathrm{CAF1}^{-/}$ mice challenged with LPS compared to the wild-type mice with LPS stimulation. As for ICAM-1, LPS stimulation greatly increased ICAM-1 expression in both wild-type and $\mathrm{CAF1}^{-/-}$mice compared to NS stimulation, and the ICAM-1 levels in $\mathrm{CAF1}^{-/-}$mice were significantly higher than those in wild-type mice (Figure 4B). 


\section{IL-8 and ICAM-I mRNA expression in wild-type and $\mathrm{CAFI}^{-I^{-}}$mice}

Next, we determined the effect of CAF1 deficiency on IL-8 and ICAM-1 expression at RNA level. Wild-type and $C A F 1^{-/-}$mice were treated with NS or LPS for 8 hours, and then the total RNA of lung tissues was extracted and IL-8 and ICAM-1 mRNA levels were measured using real-time PCR. Figure 5A shows that IL-8 mRNA levels in wild-type mice with NS group was comparable to that in $\mathrm{CAF1}^{-/-}$mice with NS group. In accordance with the results obtained for proteins, IL-8 mRNA levels in $\mathrm{CAF1}^{-/-}$mice with LPS stimulation were significantly higher than those in wild-type mice with LPS stimulation. ICAM-1 mRNA results were similar to the results obtained for proteins - ICAM-1 mRNA levels increased after LPS stimulation in both wild-type and $\mathrm{CAF1}^{-/-}$mice compared to NS stimulation. ICAM-1 mRNA

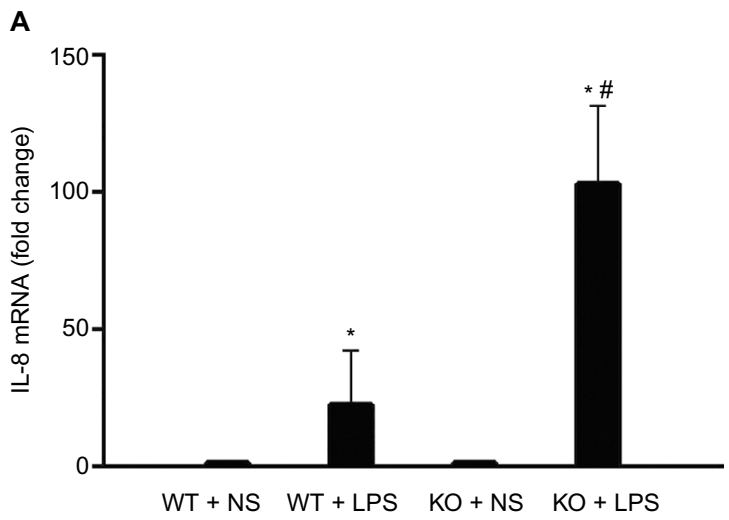

B

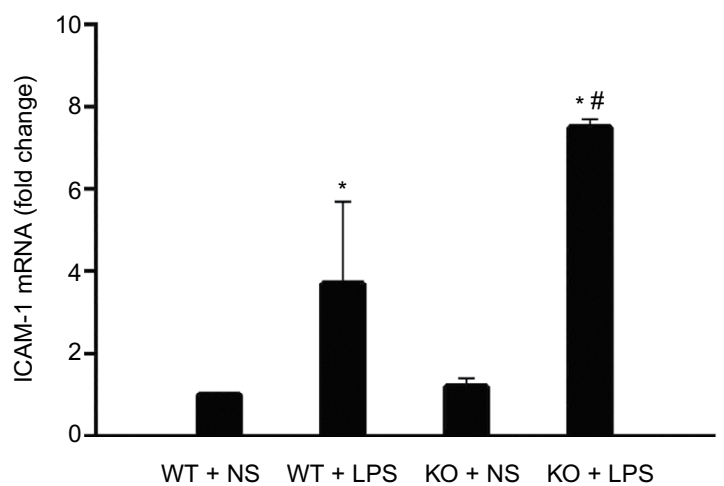

Figure 5 CAFI knockout influences LPS-induced IL-8 and ICAM-I mRNA expression. IL-8 and ICAM-I mRNA levels in wild-type mice were comparable to those in $\mathrm{CAFI-1-}^{-1}$ mice after NS stimulation and were greatly increased in both wildtype and $\mathrm{CAFI}^{-1-}$ mice following LPS stimulation (A and B). IL-8 mRNA levels in CAFI-1- mice challenged with LPS were significantly higher than those in wild-type mice (A). ICAM-I mRNA levels in CAFI-1- mice following LPS stimulation were significantly more than those in wild-type mice (B). The data are expressed as mean \pm SEM. $n=3, * P<0.05$ vs NS, $\# P<0.05$ vs wild-type mice. An independent sample $t$-test was used.

Abbreviations: WT, wild-type mice; KO, CAFI-knockout mice; NS, normal saline; LPS, lipopolysaccharide; ICAM-I, intercellular adhesion molecule-I; IL-8, interleukin-8; SEM, standard error of the mean. levels in $\mathrm{CAF1}^{-1^{-}}$mice with LPS stimulation were greatly higher than those in wild-type mice with LPS stimulation (Figure 5B).

\section{Discussion}

Acute lung injury is characterized as acute lung inflammation with PMN adhesion, accumulation, and infiltration into the lung tissue. ${ }^{9}$ Both ICAM-1 and IL-8 play important roles during acute lung injury. ${ }^{12}$

Our previous study showed that $C A F 1$ is involved in the regulation of ICAM-1 and IL-8 expression in human pulmonary microvascular endothelial cells in vitro. Whether CAF 1 could exert its effect on ICAM-1 and IL-8 expression in vivo and eventually influence acute lung injury remain unclear. In the present study, we showed that LPS stimulation led to higher IL-8 and ICAM-1 expression and more severe lung histological injury in $\mathrm{CAF1}^{-/-}$mice compared to the wild-type mice, which reveals an important role for $C A F 1$ in LPS-stimulated IL-8 and ICAM-1 expression in vivo and affects the progression of acute lung injury.

CAF1 is a subunit of the CCR4-NOT complex and has been reported to be required for the degradation of mRNA with ARE at $3^{\prime}$-UTR. ${ }^{3}$ Considering that both ICAM-1 and IL-8 mRNAs have ARE at $3^{\prime}$-UTR, we examined ICAM-1 and IL-8 expression in $\mathrm{CAF1}^{-/-}$and wild-type mice at mRNA and protein level. The results showed that IL-8 and ICAM-1 mRNA and protein levels in $\mathrm{CAFI}^{-/^{-}}$mice with NS stimulation were comparable to those in wild-type mice. IL-8 and ICAM-1 levels in $\mathrm{CAF1}^{-\gamma^{-}}$mice stimulated with LPS were significantly higher than those in wild-type mice with LPS stimulation. These results in vivo are not completely the same as the results in vitro obtained in our previous study. ${ }^{7}$ In brief, $C A F 1$ deficiency did not influence IL- 8 and ICAM-1 expression in the intact mice but significantly increased IL-8 and ICAM-1 levels after LPS stimulation compared to the wild-type mice. We postulate that other factors (such as CNOT8) in mice may compensate for $C A F 1$ deficiency; however, LPS stimulation inactivates those factors. A previous study reported that both CAF1 and CNOT8 are ubiquitously expressed in adult mouse tissues, ${ }^{13}$ and another study showed $C A F 1$ and CNOT8 have overlapping roles in vitro. ${ }^{14}$ Whether CNOT8 compensates $C A F 1$ in vivo has not been completely known. Recently, Maryati et a ${ }^{15}$ reported that $C A F 1$ and CCR 4 are both required for deadenylation by the human CCR4-NOT nuclease module. Whether CCR4 is necessary for RNA degradation in mice also remains unclear. Considering the critical roles of IL-8 and ICAM-1 in acute lung injury, ${ }^{16,17}$ LPS-induced significant increase in IL-8 and 


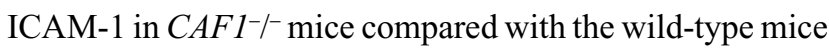
could lead to more severe lung inflammation and lung injury, which is in accordance with the histological results.

Previous studies reported that $\mathrm{CAFI}^{-/}$mice have abnormal spermatogenesis and increased bone mass. ${ }^{18,19}$ In the present study, we showed that $\mathrm{CAFI}^{-/-}$mice demonstrated more severe LPS-induced acute lung injury, which was probably due to increased IL-8 and ICAM-1 expression. Whereas, whether abnormal spermatogenesis or increased bone mass was involved in inflammation remains unknown. The present study, together with the previous studies, showed that $C A F 1$ is a multifunctional protein. The upstream and downstream components of $C A F 1$ and the factors that compensate for it are poorly understood. Further studies are needed to answer these questions.

\section{Conclusion}

The present results indicate that LPS stimulation led to higher IL-8 and ICAM-1 expression and more severe lung histological injury in $C A F 1$-knockout mice compared to the wild-type mice, which reveals an important role for $C A F 1$ in LPS-stimulated IL-8 and ICAM-1 expression in vivo and affects the progression of acute lung injury.

\section{Acknowledgments}

This study was supported by the National Natural Science Foundation of China (Grant No 81300052), the Natural Science Foundation of Jiangsu Province (Grant No BK20130402), the China Postdoctoral Science Foundation (Grant No 2015M570420), Jiangsu Provincial Health and Family Planning Commission Science Foundation (Grant No H201558), and Lianyungang Science and Technology Bureau Foundation (Grant No SH1401).

\section{Disclosure}

The authors report no conflicts of interest in this work.

\section{References}

1. Collart MA, Panasenko OO. The Ccr4-not complex. Gene. 2012; 492(1):42-53.
2. Tucker M, Valencia-Sanchez MA, Staples RR, Chen J, Denis CL, Parker R. The transcription factor associated Ccr4 and Caf1 proteins are components of the major cytoplasmic mRNA deadenylase in Saccharomyces cerevisiae. Cell. 2001;104(3):377-386.

3. Schwede A, Ellis L, Luther J, Carrington M, Stoecklin G, Clayton C. A role for Caf1 in mRNA deadenylation and decay in trypanosomes and human cells. Nucleic Acids Res. 2008;36(10):3374-3388.

4. Fan J, Heller NM, Gorospe M, Atasoy U, Stellato C. The role of posttranscriptional regulation in chemokine gene expression in inflammation and allergy. Eur Resp J. 2005;26(5):933-947.

5. Ogilvie RL, Sternjohn JR, Rattenbacher B, et al. Tristetraprolin mediates interferon-gamma mRNA decay. $J$ Biol Chem. 2009;284(17):11216-11223.

6. Shi JX, Su X, Xu J, Zhang WY, Shi Y. MK2 posttranscriptionally regulates TNF-alpha-induced expression of ICAM-1 and IL-8 via tristetraprolin in human pulmonary microvascular endothelial cells. Am J Physiol Lung Cell Mol Physiol. 2012;302(8):L793-L799.

7. Shi JX, Li JS, Hu R, et al. CNOT7/hCAF1 is involved in ICAM-1 and IL-8 regulation by tristetraprolin. Cell Signal. 2014;26(11):2390-2396.

8. Sandler H, Kreth J, Timmers HT, Stoecklin G. Not1 mediates recruitment of the deadenylase Caf1 to mRNAs targeted for degradation by tristetraprolin. Nucleic Acids Res. 2011;39(10):4373-4386.

9. Bhatia M, Moochhala S. Role of inflammatory mediators in the pathophysiology of acute respiratory distress syndrome. J Pathol. 2004;202(2):145-156.

10. Wang H, Yang H, Shivalila CS, et al. One-step generation of mice carrying mutations in multiple genes by CRISPR/Cas-mediated genome engineering. Cell. 2013;153(4):910-918.

11. Kabir K, Gelinas JP, Chen M, et al. Characterization of a murine model of endotoxin-induced acute lung injury. Shock. 2002;17(4):300-303.

12. Pober JS, Cotran RS. Cytokines and endothelial cell biology. Physiol Rev. 1990;70(2):427-451.

13. Chen C, Ito K, Takahashi A, et al. Distinct expression patterns of the subunits of the CCR4-NOT deadenylase complex during neural development. Biochem Biophys Res Commun. 2011;411(2):360-364.

14. Aslam A, Mittal S, Koch F, Andrau JC, Winkler GS. The Ccr4-NOT deadenylase subunits CNOT7 and CNOT8 have overlapping roles and modulate cell proliferation. Mol Biol Cell. 2009;20(17):3840-3850.

15. Maryati M, Airhihen B, Winkler GS. The enzyme activities of Caf1 and Ccr4 are both required for deadenylation by the human Ccr4-Not nuclease module. Biochem J. 2015;469(1):169-176.

16. Shen Y, Wang D, Wang X. Role of CCR2 and IL-8 in acute lung injury: a new mechanism and therapeutic target. Expert Rev Resp Med. 2011;5(1):107-114.

17. Bird MD, Morgan MO, Ramirez L, Yong S, Kovacs EJ. Decreased pulmonary inflammation after ethanol exposure and burn injury in intercellular adhesion molecule-1 knockout mice. J Burn Care Res. 2010;31(4):652-660.

18. Berthet C, Morera AM, Asensio MJ, et al. CCR4-associated factor CAF1 is an essential factor for spermatogenesis. Mol Cell Biol. 2004;24(13):5808-5820.

19. Washio-Oikawa K, Nakamura T, Usui M, et al. Cnot7-null mice exhibit high bone mass phenotype and modulation of BMP actions. $J$ Bone Miner Res. 2007;22(8):1217-1223.
Journal of Inflammation Research

\section{Publish your work in this journal}

The Journal of Inflammation Research is an international, peer-reviewed open access journal that welcomes laboratory and clinical findings on the molecular basis, cell biology and pharmacology of inflammation including original research, reviews, symposium reports, hypothesis formation and commentaries on: acute/chronic inflammation; mediators of
Dovepress

inflammation; cellular processes; molecular mechanisms; pharmacology and novel anti-inflammatory drugs; clinical conditions involving inflammation. The manuscript management system is completely online and includes a very quick and fair peer-review system. Visit http://www.dove press.com/testimonials.php to read real quotes from published authors. 